\title{
Atuação de profissionais de saúde para amamentação na primeira hora: Revisão
}

\section{integrativa}

\author{
Performance of health professionals for breastfeeding in the first hour: Integrative review \\ Desempeño de los profesionales de la salud para la lactancia materna em la por primera hora: \\ Revisión integrativa
}

Recebido: 19/02/2021 | Revisado: 27/02/2021 | Aceito: 03/03/2021 | Publicado: 10/03/2021

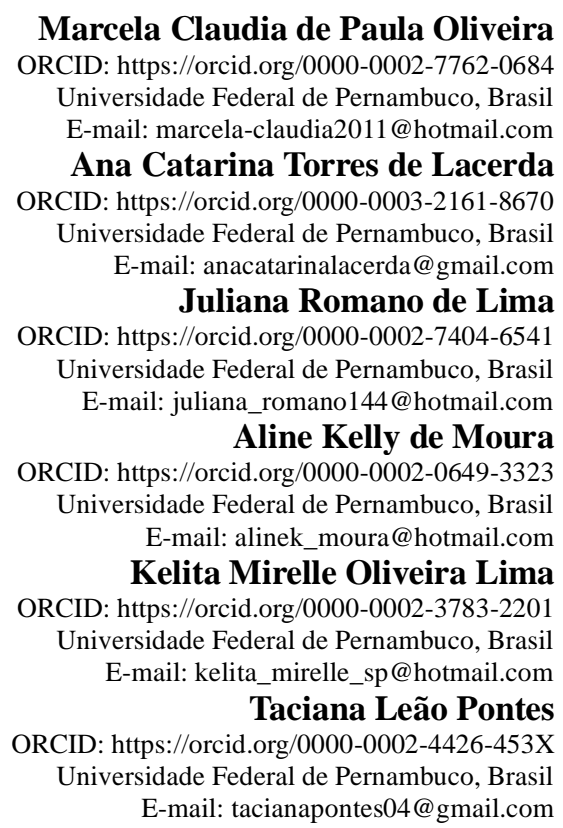

\begin{abstract}
Resumo
Objetivo: Este estudo objetivou identificar a atuação de profissionais de saúde no aleitamento materno exclusivo na primeira hora de vida do recém-nascido. Método: revisão integrativa da literatura, pautada na pergunta de pesquisa: Qual o conhecimento científico produzido na literatura nacional e internacional sobre a atuação de profissionais de saúde no aleitamento materno exclusivo na primeira hora de vida do recém-nascido? A busca das publicações científicas ocorreu nas bases de dados BDENF, CINAHL, LILACS, MEDLINE/PUBMED, SCOPUS e a biblioteca virtual de saúde SciELO, utilizando os descritores: profissional de saúde, Aleitamento materno, recém-nascido e Relações mãe-filho. Após realizar o cruzamento e recorte temporal dos últimos dez anos (do ano de 2010 ao de 2020), as buscas se deram em julho a janeiro de 2021 e foram encontradas 4.015 publicações, no qual foram excluídos 3.543 e analisados 472 resumos, tendo como amostra final oito artigos. Resultados a amostra final foi composta por 08 artigos e identificou as principais atuações dos profissionais de saúde ao aleitamento materno exclusivo na primeira hora: aconselhamento no pré-natal e apoio verbal após o parto. Os fatores limitadores dessa prática foram à assistência restrita às normas da instituição hospitalar, o parto cesáreo e o excesso de trabalho. Conclusão: Para o apoio e incentivo às mulheres da amamentação na primeira hora de vida do recém-nascido é imprescindível à atualização teórico prática do profissional de saúde para enfrentar suas fragilidades.
\end{abstract}

Palavras-chave: Profissional da saúde; Aleitamento materno; Recém-nascido; Relações mãe-filho.

\begin{abstract}
Objective: This study aimed to identify the role of health professionals in exclusive breastfeeding in the first hour of life of the newborn. Method: Integrative literature review, based on the research question: What is the scientific knowledge produced in national and international literature on the role of health professionals in exclusive breastfeeding in the first hour of life of the newborn? The search for scientific publications occurred in the databases BDENF, CINAHL, LILACS, MEDLINE / PUBMED, SCOPUS and the virtual health library SciELO, using the descriptors: health professional, breastfeeding, newborn and mother-child relationships. After performing the crossing
\end{abstract}


and temporal cut of the last ten years (from 2010 to 2020), the searches took place in July to January 2021 and 4,015 publications were found, in which 3,543 were excluded and 472 abstracts were analyzed, taking as a sample final eight articles. Results: the final sample consisted of 08 articles and identified verbal orientation in the prenatal and after delivery as the main professional performance. The limiting factors of this practice were assistance restricted to the rules of the hospital, cesarean delivery and overwork. Conclusion: For the support and encouragement of breastfeeding women in the first hour of life of the newborn, it is essential to update the theoretical and practical health professional to face their weaknesses.

Keywords: Health professional; Breastfeeding; Newborn; Mother-child relations.

\section{Resumen}

Objetivo: Este estudio tuvo identificar el papel de los profesionales de la salud en la lactancia materna exclusiva en la primera hora de vida del recién nacido. Método: revisión integradora de la literatura, a partir de la pregunta de investigación: ¿Cuál es el conocimiento científico producido en la literatura nacional e internacional sobre el papel de los profesionales de la salud en la lactancia materna exclusiva en la primera hora de vida del recién nacido? La búsqueda de publicaciones científicas se realizó en las bases de datos BDENF, CINAHL, LILACS, MEDLINE / PUBMED, SCOPUS y la biblioteca virtual de salud SciELO, utilizando los descriptores: profesional de la salud, lactancia materna, recién nacido y relaciones madre-hijo. Luego de realizar el cruzamiento y corte temporal de los últimos diez años (de 2010 a 2020), las búsquedas se realizaron en julio a enero de 2021 y se encontraron 4.015 publicaciones, de las cuales se excluyeron 3.543 y se analizaron 472 resúmenes, tomando como muestra final. ocho artículos. Resultados: La muestra final constó de 08 artículos e identificaron las principales acciones de los profesionales de la salud ante la lactancia materna exclusiva en la primera hora: consejería prenatal y apoyo verbal posparto. Los factores limitantes de esta práctica fueron la asistencia restringida a las reglas del hospital, el parto por cesárea y el exceso de trabajo. Conclusión: Para el apoyo y estímulo de las mujeres que amamantan en la primera hora de vida del recién nacido, es fundamental la actualización teórica y práctica del profesional de la salud para enfrentar sus debilidades.

Palabras clave: Profesional de la salud; Lactancia materna; Recién nacido; Relaciones madre-hijo.

\section{Introdução}

A principal iniciativa global para a promoção do aleitamento materno em serviços materno infantis é a Iniciativa Hospital Amigo da Criança (IHAC) e os 10 Passos para o sucesso da amamentação, lançada em 1991 pelo Fundo das Nações Unidas para a Infância (UNICEF) e a Organização Mundial da Saúde (OMS) adotados por mais de 156 países (Matos et al. 2010; Esteves et al. 2014) e a Rede Cegonha (2011).

Dentre os "Dez passos para o sucesso do aleitamento materno", o quarto passo versa sobre ajudar as mães a iniciar o aleitamento materno na primeira meia hora após o nascimento, colocando o bebê em contato pele a pele com sua mãe imediatamente após o parto por no mínimo uma hora (OMS, 2017). As primeiras horas de vida de um Recém-Nascido (RN) são consideradas um período favorável para estabelecer a amamentação e para fornecer as mães o apoio de que precisam para amamentar com sucesso (WHO, 2020). Esse contato pele a pele imediato traz como benefícios para amamentação aumentando a prevalência e duração da lactação; além de influenciar de forma positiva a relação mãe-filho. Esta é a chamada hora sagrada ou hora mágica, e de grande interação entre mãe-RN (Abdala et al. 2018).

A prática da amamentação na primeira hora de vida ainda não é um hábito incorporado a todas as rotinas hospitalares. Alguns fatores podem influenciar como: a etnia, escolaridade, idade materna, realização de pré-natal, via de parto, peso ao nascer, prematuridade e em particular as rotinas de trabalho das equipes de saúde que prestam assistência ao parto. Procedimentos de cuidados imediatos ao RN como: de aspiração de vias aéreas e a aspiração gástrica, avaliação e realização do exame físico prejudicam e prorrogam o contato precoce mãe-filho (Leite et al. 2016).

Além destes, outras práticas hospitalares dificultam o início oportuno da amamentação, como o uso de sedativos no período final do parto, a separação mãe-filho e a falta de apoio às mães na sala de parto (Thuler et al. 2018). Diferenças nos modelos de assistência implementados nas maternidades podem interferir no início precoce da amamentação, quando o poder de decisão das mães tende a ser mais limitado, tornando-as dependentes dos protocolos institucionais e profissionais envolvidos no parto (Silva et al. 2020). 
Desse modo, quando a vitalidade do recém-nascido está garantida, e for de desejo da mulher, é fundamental que o contato pele a pele ocorra. Para tal faz-se necessário um ambiente tranquilo, que a equipe de saúde auxilie a mãe a posicionarse confortavelmente, estejam atentos ao estado de alerta e procura do bebê pelo seio. Os profissionais devem evitar manobras que apressem o bebê na amamentação buscando favorecer a confiança materna, assistindo o binômio de modo humanizado e corroborando com as boas práticas obstétricas (Pernambuco, 2015; Leite et al. 2016).

A prevalência de aleitamento materno na primeira hora de vida, no Brasil, ainda é considerada baixa, cerca de 67,7\% (Antunes et al. 2017), o que indica a necessidade de ações governamentais, institucionais e dos profissionais de saúde para a melhoria dessa taxa. Pois, sabe-se que estes exercem papel fundamental na concretização da amamentação na primeira hora de vida (Antunes et al. 2017; Silva et al. 2020).

Os profissionais de saúde desempenham um papel importante na promoção da amamentação entre as mães. A promoção dessa prática para ser bem sucedida depende de seus conhecimentos, atitudes, motivações e habilidades de comunicação. Embora esperemos profissionais de saúde incluindo médicos, enfermeiras e vários outros profissionais estar bem informados sobre a amamentação nem sempre isto acontece. Muitos profissionais de saúde têm baixo nível de confiança, competência e conhecimento da prática para amamentação (Al-Nuaimi, Ali \& Hatem Ali, 2019).

Assim, o conhecimento e treinamento inadequados entre os profissionais de saúde são considerados barreiras para auxiliar as mães. No entanto, as evidências também sugerem que o conhecimento e o apoio de profissionais saúde bem treinados, especialmente enfermeiras e parteiras, aumenta taxas de início e continuação da amamentação, além de reduzir a mortalidade neonatal em 22\% (Gavine et al. 2017).

Considerando a importância da amamentação na primeira hora de vida para a saúde infantil e materna e sucesso da sua manutenção, sabendo dos desafios para a sua plena incorporação pela equipe de saúde que atuam na assistência ao parto, considerando que pode existir o despreparo e a falta de sensibilização de alguns profissionais para promover este momento, justifica-se estudos que elucidem junto à literatura qual percepção destes acerca dessa prática. Este Estudo objetiva identificar a atuação de profissionais de saúde no aleitamento materno exclusivo na primeira hora de vida do recém-nascido.

\section{Metodologia}

Trata-se de uma revisão integrativa da literatura. Esse tipo de revisão tem como finalidade reunir, e resumir o conhecimento científico, antes produzido sobre o tema investigado. Avalia, sintetiza e busca nas evidências disponíveis a contribuição para o desenvolvimento da temática (Galvão et al. 2010).

Para o desenvolvimento deste estudo, foram seguidas seis etapas: 1- identificação do tema e da pergunta norteadora; 2- estabelecimento de critérios para inclusão e exclusão dos estudos e coleta de dados; 3- categorização das informações extraídas dos estudos incluídos; 4- Análise crítica dos estudos incluídos; 5- Discussão dos resultados; 6- Apresentação da revisão integrativa (Mendes, Silveira \& Galvão, 2008).

Para responder a pergunta norteadora desta pesquisa: Qual o conhecimento científico produzido na literatura nacional e internacional sobre a atuação de profissionais de saúde no aleitamento materno exclusivo na primeira hora de vida do recémnascido? Foram realizadas buscas, de julho a janeiro de 2021, em fontes nacionais e internacionais, por meio do Portal CAPES (Banco de Bases de Dados da Coordenação de Aperfeiçoamento de Pessoal de Nível Superior), sendo selecionadas as seguintes bases de dados: Medical Literature Analysis and Retrieval System Online (MEDLINE via PubMed), Literatura Latino-Americana e do Caribe em Ciências da Saúde (LILACS), SCOPUS, The Cumulative Index to Nursing and Allied Health Literature (CINAHL), e a biblioteca eletrônica Scientific Electronic Library Online (SciELO). Também sendo consultada a base Banco de Dados em Enfermagem (BDENF). 
A estratégia para identificação dos artigos foi realizada através de termos controlados e não controlados na base de Descritores em Ciências da Saúde (DeCS) utilizando a combinação dos descritores "Profissional da saúde”, "Aleitamento Materno" e "recém-nascido" ou "relações mãe-filho", todos incluídos nos Descritores em Ciências da Saúde (DeCS) e suas respectivas traduções padronizadas no Medical Subject Heading (MESH): "Health Professional", "Breastfeeding", "Newborn", "Mother-Child Relations". Foram realizados os cruzamentos, utilizando os operadores booleanos and entre os descritores: Profissional da saúde and recém-nascido; profissional da saúde and aleitamento materno; profissional da saúde and relações mãe-filho (Quadro 1).

Quadro 1. Publicações encontradas a partir do cruzamento dos descritores, segundo bases de dados.

\begin{tabular}{|c|c|c|c|c|}
\hline $\mathrm{C}_{\text {Base }}^{\text {Ce Dados }}$ & 1 & 2 & 3 & Total \\
\hline BDENF & 33 & 61 & 5 & 99 \\
\hline LILACS & 254 & 159 & 6 & 419 \\
\hline SciELO & 68 & 39 & 7 & 114 \\
\hline PUBMED & 380 & 611 & 439 & 1.430 \\
\hline CINAHL & 312 & 465 & 193 & 970 \\
\hline SCOPUS & 443 & 339 & 201 & 983 \\
\hline Total & 1.490 & 1.674 & 851 & 4.015 \\
\hline
\end{tabular}

1. Profissional da saúde/Health professional AND Aleitamento materno/Breastfeeding

2. Profissional da saúde/Health professional AND Recém-nascido/Newborn

3. Profissional de saúde/Health professional AND Relações mãe-filho/Mother-child relations

Fonte: Autores.

Para selecionar os artigos, foram adotados os seguintes critérios de inclusão: artigos nacionais e internacionais, disponíveis online de forma gratuita e na íntegra, em português, inglês e espanhol, que respondessem à questão norteadora da pesquisa e que estivessem no recorte temporal dos últimos dez anos (do ano de 2010 ao de 2020) e com a presença de pelo menos dois dos descritores descritos no título. Como critérios de exclusão: artigos de revisão, capítulos de livros, editoriais, artigos de validação, relato de casos, dissertações, teses e artigos em duplicidade. Estudos repetidos foram considerados apenas uma vez e computados na base de dados com o maior número de artigos.

Após os cruzamentos, foram encontradas 4.015 publicações. Para seguimento do método da revisão, foram considerados apenas os estudos provenientes do cruzamento dos descritores. Os títulos desses 4.015 estudos foram lidos, sendo realizado o julgamento se cada um respondia à pergunta norteadora e se estava inserido nos demais critérios de inclusão, destes 472 resumos foram analisados. Nos casos em que houve dúvida sobre o conteúdo do estudo, este foi selecionado para leitura na íntegra, sendo pré-selecionados. Dos 87 artigos lidos na íntegra, 40 trabalhos eram do tipo revisão, 24 relatos de experiência, 7 editoriais, 4 teses, 4 dissertações. Assim, 8 pesquisas constituíram o corpus final da revisão. O processo de busca e seleção dos estudos para composição da amostra está esquematizado na Figura 1. 
Figura 1. Processo de seleção das publicações sobre o conhecimento científico produzido na literatura sobre a percepção de profissionais de saúde e amamentação na primeira hora. Recife-PE, Brasil. 2020.
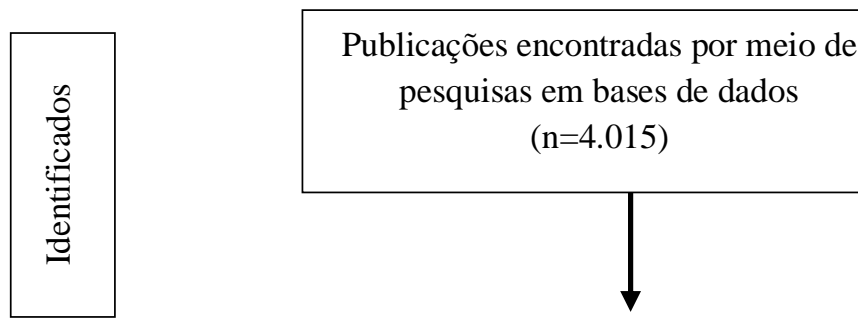
pesquisas em bases de dados $(\mathrm{n}=4.015)$

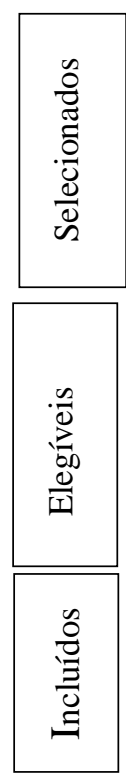

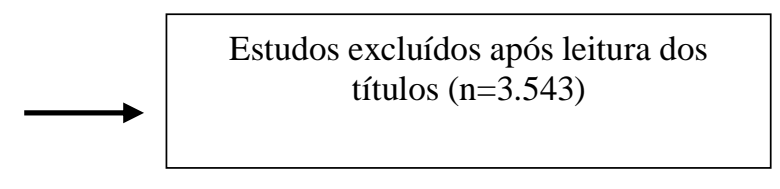
títulos $(\mathrm{n}=3.543)$

Fonte: Preferred Reporting Items for Systematic Reviews and Meta-Analyses the PRISMA.

O rigor metodológico dos estudos selecionados foi avaliado por meio de um instrumento adaptado do Critical Appraisal Skills Programme (CASP, 2013), que classifica os estudos como: de boa qualidade metodológica e viés reduzido (categoria A - 6 a 10 pontos) e com qualidade metodológica satisfatória (categoria B - no mínimo 5 pontos). O instrumento de avaliação possui dez itens, analisando as publicações quanto à pergunta norteadora, busca na literatura, delimitação dos critérios de inclusão e exclusão, busca e seleção dos estudos, coleta de dados, avaliação dos estudos, apresentação e interpretação dos resultados, descrição das etapas percorridas e benefícios.

Para a realização da coleta de dados, foi utilizado um instrumento adaptado e validado pelas autoras que contemplam as principais informações das publicações selecionadas. Este instrumento contém os seguintes dados identificação da publicação, objetivo do estudo, método, nível de evidência, atuação profissional, resultados e conclusões (Pompeo, Rossi \& Galvão, 2009). As pesquisas incluídas na revisão foram analisadas quanto ao nível de evidência científica segundo Oxford Centre for Evidence-based Medicine (2009). Nesse critério, a evidência é classificada em 1A (Revisão sistemática de ensaios clínicos controlados randomizados), 1B (Ensaio clínico controlado randomizado com intervalo de confiança estreito), 1C (Resultados terapêuticos do tipo "tudo ou nada"), 2A (Revisão Sistemática de Estudos de Coorte), 2B (Estudo de Coorte), 2C (Observação de resultados terapêuticos/ Estudo Ecológico), 3A (Revisão Sistemática de Estudos Caso-Controle), 3B (Estudo Caso-Controle), 4 (Relatos de Caso) e 5 (Opinião de especialistas desprovida de avaliação crítica ou baseada em matérias básicas) (Oxford, 2009). 


\section{Resultados}

Dos oito artigos selecionados, dois foram extraídos da SciELO, um na BDENF, três na PUBMED, um na CINAHL, um na SCOPUS e na base de dados da LILACS não foram encontrados artigos. Os estudos foram publicados entre 2012 a 2020, em português e inglês. Quanto ao tipo de delineamento de pesquisa dos estudos avaliados, dois artigos são descritivos (Antunes et al. 2017; Rodrigues et al. 2020), dois qualitativos (Kologeski et al. 2017; Fassarella et al. 2018), um experimental (Nuaimi et al. 2019) e três transversal (Vieira et al. 2010; Pereira et al. 2013; Beyene et al. 2019).

$\mathrm{Na}$ avaliação do rigor metodológico dos artigos selecionados, foi utilizado o CASP, no qual são enquadrados como de nível "A" os artigos que alcançam a pontuação acima de seis e com qualidade metodológica satisfatória e os de nível B com pontuação de no mínimo cinco, para esta revisão foram encontrados artigos de categoria 2C (Observação de resultados terapêuticos/ Estudo Ecológico).

A forma principal de atuação dos profissionais vista nos estudos $(n=8)$, foram à orientação verbal as mulheres: seja no pré-natal ou no pós-parto. Observou-se que as mães que iniciaram a amamentação na primeira hora após o nascimento receberam essa orientação durante a realização do pré-natal e no hospital no período do pós-parto. Esta ação pela equipe contribuiu para o início do aleitamento materno na primeira hora de vida (Pereira et al. 2013; Vieira et al. 2010). A prática do contato pele a pele logo após o nascimento e colocavam a criança no seio da mãe pelos profissionais de saúde também foi identificada, porém em apenas um estudo (Beyene et al. 2019).

O conhecimento da equipe de enfermagem acerca da amamentação na primeira hora foi considerado insatisfatório em alguns estudos. Outras dificuldades para efetivação dessa prática foi: deficiência no conhecimento teórico prático dos profissionais sobre a amamentação de um modo geral, normas hospitalares engessadas, parto cesáreo e o excesso de trabalho (Kologeski et al. 2017; Fassarella et al. 2018; Rodrigues et al. 2020).

Contudo, prática da amamentação na primeira hora teve como elemento facilitador a ação educativa em formato de workshop, melhorando a atuação prática dos profissionais após participação do mesmo (NUAIMI et al. 2019).

Os profissionais que compuseram as amostras dos estudos foram: enfermeiros, parteiras, auxiliares e técnicos de enfermagem, médicos pediatras e gineco-obstetras, nutricionistas, residentes de pediatria/obstetrícia (Vieira et al. 2010; Pereira et al. 2013; Antunes et al. 2017; Kologeski et al. 2017; Fassarella et al. 2018; Nuaimi et al. 2019; Beyene et al. 2019; Rodrigues et al. 2020). As sínteses dos artigos estão apresentados no Quadro 2.

Quadro 2. Distribuição das publicações incluídas na revisão integrativa de acordo com autoria, ano de publicação, base de dados, objetivo, método e níveis de evidência. Recife-PE, Brasil. 2020.

\begin{tabular}{|c|c|c|c|c|c|}
\hline $\begin{array}{l}\text { Autores/ } \\
\text { Ano/ } \\
\text { Base de } \\
\text { dados }\end{array}$ & Objetivo & $\begin{array}{l}\text { Método/ Nível de } \\
\text { Evidência }\end{array}$ & Resultados & $\begin{array}{l}\text { Forma de } \\
\text { atuação }\end{array}$ & Conclusões \\
\hline $\begin{array}{l}\text { Vieira et al. } \\
2010 . \\
\text { PUBMED }\end{array}$ & $\begin{array}{l}\text { Avaliar a prevalência do } \\
\text { início da amamentação } \\
\text { na primeira hora de vida } \\
\text { em Feira de Santana, } \\
\text { Bahia, Brasil, entre } \\
2004 \text { e } 2005 \text {, e } \\
\text { investigar a influência } \\
\text { da maternidade, da } \\
\text { criança e do pré-natal } \\
\text { nesta prática. }\end{array}$ & $\begin{array}{l}\begin{array}{l}\text { Estudo transversal } \\
\text { extraído dos resultados } \\
\text { de uma coorte } \\
\text { contemporânea. }\end{array} \\
\text { Amostra: Puérperas } \\
\text { Nível de evidência: 2C }\end{array}$ & $\begin{array}{l}\text { As mães iniciaram a } \\
\text { amamentação na } \\
\text { primeira hora após o } \\
\text { nascimento. O início } \\
\text { precoce da amamentação } \\
\text { foi associado ao } \\
\text { nascimento em gestação } \\
\text { a termo. }\end{array}$ & $\begin{array}{l}\text { Orientação } \\
\text { verbal no pré- } \\
\text { natal. }\end{array}$ & $\begin{array}{l}\text { Os profissionais de } \\
\text { saúde devem favorecer } \\
\text { e estimular essa prática } \\
\text { durante a orientação no } \\
\text { pré-natal. }\end{array}$ \\
\hline
\end{tabular}




\begin{tabular}{|c|c|c|c|c|c|}
\hline $\begin{array}{l}\text { Pereira et al. } \\
2013 \text {. }\end{array}$ & $\begin{array}{l}\text { Investigar como o passo } \\
4 \text { da Iniciativa Hospital } \\
\text { Amigo da Criança foi } \\
\text { aplicado, avaliar a } \\
\text { prevalência } \\
\text { amamentação da } \\
\text { primeira hora após o } \\
\text { nascimento e analisar os } \\
\text { fatores associados à não } \\
\text { amamentação neste } \\
\text { período de vida. }\end{array}$ & $\begin{array}{l}\text { Estudo transversal. } \\
\text { Amostra: Puérperas } \\
\text { Nível de evidência: } 2 \mathrm{C}\end{array}$ & $\begin{array}{l}\text { A prevalência de } \\
\text { amamentação na } \\
\text { primeira hora em } \\
\text { mulheres que receberam } \\
\text { orientação no pré-natal e } \\
\text { no período do pós-parto } \\
\text { foi satisfatória. }\end{array}$ & $\begin{array}{l}\text { Orientação } \\
\text { verbal: no } \\
\text { pré-natal e } \\
\text { pós-parto. } \\
\text { Colocação do } \\
\text { bebê no seio } \\
\text { da mãe. }\end{array}$ & $\begin{array}{l}\text { A ajuda prestada pela } \\
\text { equipe de saúde à } \\
\text { amamentação ao } \\
\text { nascimento, a } \\
\text { realização de pré-natal, } \\
\text { o parto normal e o } \\
\text { peso adequado ao } \\
\text { nascer contribuíram } \\
\text { para o início do } \\
\text { aleitamento materno na } \\
\text { primeira hora de vida. }\end{array}$ \\
\hline $\begin{array}{l}\text { Antunes et } \\
\text { al. } 2017 \text {. } \\
\text { BVS }\end{array}$ & $\begin{array}{l}\text { Verificar } 0 \\
\text { conhecimento e prática } \\
\text { sobre a amamentação na } \\
\text { primeira hora de vida } \\
\text { entre membros da } \\
\text { equipe multiprofissional } \\
\text { de um hospital do } \\
\text { município de Maringá, } \\
\text { Paraná. }\end{array}$ & $\begin{array}{l}\begin{array}{l}\text { Estudo descritivo- } \\
\text { exploratório com } \\
\text { abordagem qualitativa. }\end{array} \\
\text { Profissionais: } \\
\text { enfermeiros, auxiliares } \\
\text { em enfermagem, } \\
\text { técnicos em } \\
\text { enfermagem, pediatras, } \\
\text { gineco-obstetras, } \\
\text { nutricionistas, } \\
\text { residentes em pediatria } \\
\text { ou obstetrícia. } \\
\text { Nível de Evidência: } 2 \mathrm{C}\end{array}$ & $\begin{array}{l}\text { Deficiência no } \\
\text { conhecimento dos } \\
\text { profissionais sobre a } \\
\text { amamentação na } \\
\text { primeira hora de vida, } \\
\text { carência de preparo da } \\
\text { equipe, necessidade de } \\
\text { capacitação; além disso, } \\
\text { a prática não acontece na } \\
\text { instituição. }\end{array}$ & $\begin{array}{l}\text { Orientação } \\
\text { verbal no pós- } \\
\text { parto. }\end{array}$ & $\begin{array}{l}\text { Sugere-se aderir } \\
\text { amamentação na } \\
\text { primeira hora de vida às } \\
\text { políticas hospitalares de } \\
\text { promoção e proteção ao } \\
\text { aleitamento materno, que } \\
\text { valorizem a equipe } \\
\text { multiprofissional e o } \\
\text { trabalho colaborativo } \\
\text { entre seus membros. }\end{array}$ \\
\hline $\begin{array}{l}\text { Kologeski } \\
\text { et al. } 2017 .\end{array}$ & $\begin{array}{l}\text { Conhecer a percepção } \\
\text { dos profissionais da } \\
\text { equipe multiprofissional } \\
\text { em relação ao contato } \\
\text { pele a pele precoce da } \\
\text { mãe com o bebê no } \\
\text { momento } \\
\text { nascimento. }\end{array}$ & 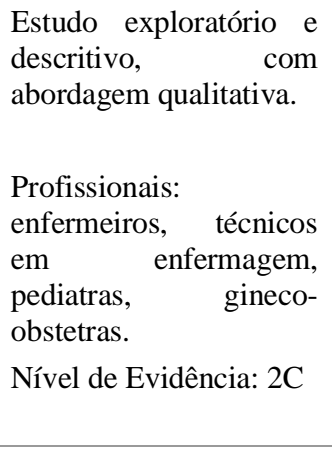 & $\begin{array}{l}\text { Emergiram cinco } \\
\text { categorias temáticas. O } \\
\text { contato pele a pele na } \\
\text { sala de parto é uma } \\
\text { prática de cuidado } \\
\text { humanizado com forte } \\
\text { evidencia para a } \\
\text { formação de vínculo } \\
\text { mãe/bebê. }\end{array}$ & $\begin{array}{l}\text { Orientação } \\
\text { verbal no pós- } \\
\text { parto. }\end{array}$ & $\begin{array}{l}\text { A adoção da } \\
\text { amamentação na } \\
\text { primeira hora de vida } \\
\text { necessita de mudança } \\
\text { de paradigmas no } \\
\text { cuidado materno e } \\
\text { neonatal no parto e } \\
\text { nascimento do modelo } \\
\text { vigente na instituição, } \\
\text { que se encontra com } \\
\text { inconformidades ao } \\
\text { recomendado. }\end{array}$ \\
\hline $\begin{array}{l}\text { Fassarella } \\
\text { et al. 2018. } \\
\text { SCOPUS }\end{array}$ & $\begin{array}{lr}\text { Compreender } & \text { a } \\
\text { percepção da equipe de } \\
\text { enfermagem acerca da } \\
\text { amamentação } \\
\text { primeira hora após o } \\
\text { nascimento do bebê. }\end{array}$ & $\begin{array}{l}\text { Estudo qualitativo de } \\
\text { abordagem descritiva. } \\
\text { Profissionais: } \\
\text { enfermeiros e técnicos } \\
\text { de enfermagem. } \\
\text { Nível de Evidência: } 2 \mathrm{C}\end{array}$ & $\begin{array}{l}\text { Foram entrevistados } 28 \\
\text { profissionais de } \\
\text { enfermagem, com idade } \\
\text { entre } 25 \text { a } 55 \text { anos, com } \\
\text { tempo de atuação entre } 1 \\
\text { a } 16 \text { anos. O melhor } \\
\text { momento para falar } \\
\text { sobre o aleitamento } \\
\text { materno na primeira hora } \\
\text { deve ser iniciado no } \\
\text { acompanhamento do pré- } \\
\text { natal. }\end{array}$ & $\begin{array}{l}\text { Orientação } \\
\text { verbal no pós- } \\
\text { parto. }\end{array}$ & $\begin{array}{l}\text { O conhecimento da equipe } \\
\text { de enfermagem acerca da } \\
\text { amamentação na primeira } \\
\text { hora foi insatisfatório e } \\
\text { essa prática precisa ser de } \\
\text { suma importância dentro } \\
\text { da unidade. }\end{array}$ \\
\hline $\begin{array}{l}\text { Nuaimi et } \\
\text { al. 2019. }\end{array}$ & $\begin{array}{l}\text { Avaliar a eficácia de um } \\
\text { programa educacional } \\
\text { sobre conhecimentos e } \\
\text { atitudes de enfermeiras } \\
\text { e parteiras sobre a } \\
\text { prática da } \\
\text { amamentação. }\end{array}$ & $\begin{array}{l}\text { Estudo experimental } \\
\text { com pré e pós-teste em } \\
\text { dois grupos. } \\
\text { Profissionais: } \\
\text { Enfermeiras e parteiras. } \\
\text { Nível de Evidência: 2C }\end{array}$ & $\begin{array}{l}\text { Foram entrevistadas } 82 \\
\text { enfermeiras e parteiras } \\
\text { foram recrutados } \\
\text { aleatoriamente para } \\
\text { realização do pré-teste } \\
\text { (antes do workshop) e } \\
\text { após } 2 \text { semanas foi } \\
\text { realizado o pós-teste } \\
\text { (depois do workshop). } \\
\text { Os resultados mostraram } \\
\text { que o workshop ajudou a } \\
\text { melhorar o } \\
\text { conhecimento e prática } \\
\text { da amamentação na }\end{array}$ & $\begin{array}{l}\text { Orientação } \\
\text { verbal no pós- } \\
\text { parto. }\end{array}$ & $\begin{array}{l}\text { A realização de um } \\
\text { workshop sobre } \\
\text { amamentação contribuiu } \\
\text { para o conhecimento e } \\
\text { práticas dos profissionais } \\
\text { para a amamentação. }\end{array}$ \\
\hline
\end{tabular}




\begin{tabular}{|c|c|c|c|c|c|}
\hline & & & primeira hora. & & \\
\hline $\begin{array}{l}\text { Beyene et } \\
\text { al. 2019. } \\
\text { PUBMED }\end{array}$ & $\begin{array}{l}\text { Avaliar os } r \text { fatores } \\
\text { associados ao término } \\
\text { precoce } \\
\text { amamentação exclusiva } \\
\text { entre as díades mãe- } \\
\text { bebê SamaraLogia- } \\
\text { Etiópia. }\end{array}$ & $\begin{array}{l}\text { Estudo transversal. } \\
\text { Amostra: puérperas } \\
\text { Nível de evidência: } 2 \mathrm{C}\end{array}$ & $\begin{array}{lrr}\text { A maioria } & \text { dos } \\
\text { entrevistados } & \text { receberam } \\
\text { orientações } & \text { sobre } \\
\text { amamentação } & \text { na } \\
\text { primeira hora no pré- } \\
\text { natal e iniciaram essa } \\
\text { prática logo após o } \\
\text { nascimento. }\end{array}$ & $\begin{array}{l}\text { Orientação } \\
\text { verbal no pré- } \\
\text { natal e no pós- } \\
\text { parto. }\end{array}$ & $\begin{array}{l}\text { As intervenções de } \\
\text { promoções a saúde } \\
\text { devem ter o objetivo } \\
\text { de educar os pais e } \\
\text { envolve-los nas } \\
\text { decisões sobre } \\
\text { amamentação } \\
\text { incentivando a prática } \\
\text { na primeira hora. }\end{array}$ \\
\hline $\begin{array}{l}\text { Rodrigues } \\
\text { et al. } 2020 .\end{array}$ & $\begin{array}{l}\text { Conhecer o perfil } \\
\text { sociodemográfico da } \\
\text { equipe multiprofissional } \\
\text { que atuam em uma } \\
\text { maternidade de risco } \\
\text { habitual e as práticas } \\
\text { atuais de amamentação } \\
\text { na primeira hora de } \\
\text { vida. }\end{array}$ & $\begin{array}{l}\text { Estudo descritivo } \\
\text { exploratório de } \\
\text { abordagem quantitativa. } \\
\text { Profissionais: } \\
\text { enfermeiros, auxiliares } \\
\text { em enfermagem, } \\
\text { técnicos } \\
\text { enfermagem, pediatras, } \\
\text { gineco-obstetras, } \\
\text { nutricionistas, } \\
\text { residentes em pediatria } \\
\text { ou obstetrícia. } \\
\text { Nível de Evidência: } 2 \mathrm{C}\end{array}$ & $\begin{array}{l}\text { A maioria dos } \\
\text { profissionais era técnica } \\
\text { de enfermagem, com } \\
\text { idade entre } 26 \text { a } 35 \text { anos, } \\
\text { com experiência } \\
\text { profissional de } 1 \text { a } 3 \\
\text { anos. O conhecimento e } \\
\text { a atuação profissional } \\
\text { para aleitamento foi } \\
\text { incompleto/ e ou } \\
\text { equivocado. }\end{array}$ & $\begin{array}{l}\text { Orientação } \\
\text { verbal no pós- } \\
\text { parto. }\end{array}$ & $\begin{array}{l}\text { Ressalta-se que para } \\
\text { obter uma cobertura } \\
\text { maior das práticas que } \\
\text { envolvem a temática é } \\
\text { necessário conhecer a } \\
\text { população que irá } \\
\text { assistir esta clientela, } \\
\text { compreender sua } \\
\text { prática clínica e alinhar } \\
\text { as iniciativas. }\end{array}$ \\
\hline
\end{tabular}

Fonte: Autores.

\section{Discussão}

O Ministério da Saúde do Brasil preconiza o aleitamento materno na primeira hora pós-parto como um dos passos a ser adotado no reconhecimento das Instituições como Hospital Amigo da Criança. Estudos têm evidenciado que o começo de tal prática até a primeira hora de vida auxilia na redução das taxas de mortalidade infantil, e corrobora para o cumprimento das metas acordadas junto à Organização das Nações Unidas (ONU) na redução da mortalidade materno-infantil, fazendo parte dos Objetivos Sustentáveis do Milênio (Brasil, 2011; ONU, 2015).

O aleitamento materno na primeira hora de vida é destacado pela OMS como um item importante para promoção, proteção e suporte à amamentação. A medida é considerada de baixo custo e de boa efetividade além de se associar a uma duração prolongada do aleitamento materno. Desse modo, os profissionais de saúde têm o papel essencial de proteger este momento sensível, pois isso vai ter repercussões para toda a vida (OMS, 2017).

Nos artigos desta revisão, a forma de atuação dos profissionais de saúde mais observada foi à orientação verbal no pré-natal ou no pós-parto (Vieira et al. 2010; Pereira et al. 2013; Antunes et al. 2017; Kologeski et al. 2017; Fassarella et al. 2018; Nuaimi et al. 2019; Beyene et al. 2019; Rodrigues et al. 2020). Existe uma associação entre amamentação na primeira hora de vida e ter recebido orientação sobre as vantagens do aleitamento materno no pré-natal, indicando que este acompanhamento favorece a preparação para amamentação.

A consulta de pré-natal destaca-se por ser onde o profissional de saúde identifica a vivência e experiência que a mulher traz consigo, e as orientações devem abranger a integralidade do cuidado, prevenção de agravos e o compromisso com a qualidade de vida do binômio (Vieira et al. 2010; Pereira et al. 2013; Beyene et al. 2019). Este acompanhamento favorece para que esta prática ocorra logo após o nascimento de forma satisfatória (Vieira et al. 2010; Pereira et al. 2013; Beyene et al. 2019).

De acordo com a OMS 2017 e o MS 2011 orientar, tanto no pré-natal quanto logo após o parto são importantes, pois no momento após o parto surgem dúvidas e problemas práticos, deixando a nutriz insegura para amamentar, então o binômio 
necessita de maior atenção. E as intervenções de promoção à saúde devem ter o objetivo de educar os pais e envolve-los nas decisões sobre amamentação incentivando a prática na primeira hora (Beyene et al. 2019).

A modalidade mais citada para auxiliar e apoiar as mães, a orientação verbal, pressupõe desconhecimento e despreparo teórico prático dos profissionais sobre a amamentação na primeira hora e seu papel para concretização da mesma. A amamentação necessita de orientações, porém não apenas de modo oral (Antunes et al. 2017; Kologeski et al. 2017; Fassarella et al. 2018; Rodrigues et al. 2020).

Variadas tecnologias da educação existem e podem contribuir, tais como: as demonstrações em manequins, vídeos, aplicativos móveis e outros recursos. As tecnologias digitais surgem para complementar à aquisição de novos conhecimentos, por serem cada vez mais utilizadas e por alcançarem a maioria dos espaços sociais (Diniz et al. 2019). No estudo realizado por Nuaimi et al. (2019), a realização de um workshop sobre amamentação ajudou a melhorar o conhecimento e prática para a amamentação na primeira hora. Essa versatilidade contribuiu para que seja reconhecido o potencial desses recursos nas práticas de saúde, favorecendo a sua incorporação de forma cada vez mais frequente (OMS, 2017).

Apenas um estudo trouxe como forma de atuação a orientação verbal e prática, auxiliando a mãe a colocar criança ao seio após o parto (Pereira et al. 2013). Considerando o pós-parto, é importante informar à mulher que é possível amamentar na sala de parto logo após o nascimento, perguntar se ela quer fazê-lo e ajudá-la nesta hora a segurar o seu bebê e a identificar se é o momento para amamentá-lo, são ações que envolvem a assistência ao recém-nascido e são reconhecidas como de significância na proteção à amamentação na primeira hora de vida (Pereira et al. 2013).

O auxílio a prática do passo 4 ainda é pouco aplicada ou realizada ainda sem uniformidade pelas equipes na assistência ao parto, mesmo em hospitais credenciados como amigo da criança. No pós-parto, a colocação do bebê no peito e a observação da pega correta pelo profissional são essenciais. A Portaria ${ }^{\circ} 371$ do Ministério da Saúde (BRASIL, 2014) estabelece que, ao nascer, o bebê seja colocado em contato imediato com a mãe, estimulando e favorecendo a primeira mamada, bem como normatiza procedimentos comprovados por estudos científicos que mostram os benefícios dessas práticas para a saúde da criança e da mulher.

Pelo menos durante a primeira hora de vida, preconiza-se adiar qualquer procedimento rotineiro de atenção ao recémnascido que separe a mãe de seu bebê, com o objetivo de permitir o contato pele-a-pele ininterrupto entre o binômio (Strapasson et al. 2011). Porém, os profissionais dos estudos, apontaram que práticas institucionais engessadas limitam o contato pele a pele e a amamentação do recém-nascido na primeira hora de vida. Ainda citaram como fatores de impedimento o parto cesariano e as analgesias (Pereira et al. 2013; Antunes et al. 2017; Kologeski et al. 2017; Antunes et al. 2017; Fassarella et al. 2018; Rodrigues et al. 2020).

O aleitamento materno exige que os profissionais dos serviços de saúde tenham disponibilidade para atender a mãe prontamente, com o risco de seu adiamento acarretar em desistência e insucesso. É fundamental que ele seja capaz de orientar e prestar auxílio no manejo do aleitamento materno com conhecimentos e habilidades clínicas. Para tal, existe a necessidade de periódicas capacitações, com vistas na sustentação de políticas e protocolos de aleitamento materno (Maroja et al. 2014; Azevedo et al. 2015).

No que diz respeito ao conhecimento e prática sobre a amamentação na primeira hora de vida entre membros da equipe multiprofissional, mostrou-se, aquém das necessidades da mulher e da criança, não assistindo a mãe que amamenta em caráter prático assistencial (Antunes et al. 2017; Kologeski et al. 2017; Rodrigues et al. 2020). A mudança de atitude do profissional de saúde, com a integração e valorização do binômio, pode facilitar a operacionalização do quarto passo da IHAC, de modo a ser realizado não apenas de forma mecanicista e fragmentada, e sim com respeito e acolhimento (D'Artibale et al. 2014; Sampaio et al. 2016). 
O processo da amamentação deve ser entendido pelo profissional como uma situação única, devendo-se adotar uma conduta de apoio e incentivo à mãe, sobretudo na primeira hora de vida do recém-nascido (Rodrigues et al. 2020).

\section{Conclusão}

Esta revisão traz como principais achados, que a atuação profissional com vistas na promoção do aleitamento na primeira hora se deu por orientações verbais no pré-natal e no período pós-parto. Observamos a existência de poucos estudos sobre o conhecimento e práticas dos profissionais que atendem a mulher e o recém-nascido no âmbito hospitalar em relação à amamentação na primeira hora de vida, porém sabe-se que estes exercem papel fundamental na concretização da amamentação na primeira hora. Também foi observada a existência de fatores limitadores para a amamentação na primeira hora de vida do recém-nascido como: tarefas rotineiras a serem cumpridas, a falta de apoio institucional e a falta de vínculo entre o profissional e a mãe. Observou-se a presença de dificuldades na adesão ampla à prática entre a equipe multiprofissional, especialmente no que se refere ao parto cesáreo. Evidenciou-se também que o modelo medicalizado e intervencionista, em relação ao parto e nascimento constitui-se como um importante balizador das inconformidades desse processo. Sendo assim, a amamentação na primeira hora de vida do recém-nascido constitui-se como um desafio, sendo, imprescindível à capacitação profissional a fim de melhorar a prática assistencial desse ato e enfrentar suas fragilidades.

Sugere-se novas produções científicas acerca da temática em questão, proporcionando assim, reflexões críticas atualizadas que enfoquem o conhecimento e práticas dos profissionais que atendem a mulher e o recém-nascido no ambiente hospitalar.

\section{Referências}

Abdala, L. G. \& Cunha, M. L. C. (2018). Contato pele a pele entre mãe e recém-nascido e amamentação na primeira hora de vida. Clinical and biomedical Research, 38(4): 356-360, 2018. https://seer.ufrgs.br/hcpa/article/view/82178

Al-Nuaimi, K., Ali, R. \& Hatem Ali, F. (2019). The effect pf a breastfeeding educational workshop on clinicians knowledge, atitudes and practices. British Journal of Midwifery, 27(4). https://www.magonlinelibrary.com/doi/abs/10.12968/bjom.2019.27.4.242

Antunes, M. B., Demitto, M. O., Soares, L. G., Radovanovic, C. A. T., Higarashi, I. H., Ichisato, S. M. T. \& Pelloso, S. M. (2017). Amamentação na primeira hora de vida: conhecimento e prática da equipe multiprofissional. Avances em Enfermagem, 35(1):19-29. http://www.scielo.org.co/pdf/aven/v35n1/v35n1a03.pdf

Azevedo, A. R. R., Alves, V. H., Souza, R. M. P., Rodrigues, D. P., Branco, M. B. L. R. \& Cruz, A. F. N. (2015). O manejo clínico da amamentação: saberes dos enfermeiros. Escola Anna Nery, 9(3), 439-445. https://www.scielo.br/pdf/ean/v19n3/1414-8145-ean-19-03-0439.pdf

Beyene, A. M., Liben, M. L. \& Arora, A. (2019). Factors associated with the early termination of exclusive breastfeeding among mother-infant dyads in SamaraLogia, Northeastern Ethiopia. BioMed Central Pediatrics, 19, 428. https://bmcpediatr.biomedcentral.com/articles/10.1186/s12887-019-1803-1

Brasil. Ministério da Saúde. Secretaria de Atenção a Saúde. (2011). Departamento de Ações Programáticas e Estratégicas. Além da sobrevivência: práticas integradas de atenção ao parto, benéficas para a nutrição e a saúde das mães e crianças. Brasília (DF): MS.

Brasil. Ministério da Saúde. Secretaria de Atenção à Saúde. (2014). Portaria no 371, de 07 de maio de 2014. Institui diretrizes para a organização da atenção integral e humanizada ao recém nascido (RN) no Sistema Único de Saúde (SUS). Diário Oficial da União. Brasília, DF.

CASP. Critical Appraisal Skills Programme. http://www.casp-uk.net/casp-tools-checklists.

D'Artibale, E. F. \& Bercini, L. O. (2014). A prática do quarto passo da iniciativa hospital amigo da criança. Revista de Enfermagem, $18(2)$, 356-364.

Diniz, C. M. M., Leal, L. P., Guedes, T. G., Linhares, F. M. P. \& Pontes, C. M. (2019). Contribuições dos aplicativos móveis para a prática do aleitamento materno: revisão integrativa. Acta Paulista de Enfermagem, 32(5). https://www.scielo.br/scielo.php?pid=S0103$21002019000500015 \&$ script=sci_abstract\&tlng=pt

Esteves, T. M. B., Daumas, R. P., Oliveira, M. I. C., Andrade, C. A. F. \& Leite, I. C. L. (2014), Fatores associados à amamentação na primeira hora de vida: revisão sistemática. Revista Saúde Pública, 48(4):697-703. http://dx.doi.org/10.1590/S0034-8910.2014048005278

Fassarella, B. P. A., Maleck, M., Ribeiro, W. A., Pimenta, É. S. S., Corrêia, M. C. B., PInheiro, D. S., Martins, L. M. \& Peixoto, M. S. B. F. (2018). Percepção da equipe de enfermagem frente ao aleitamento materno: do conhecimento à implementação. Nursing, 21 (247), $2489-2493$. https://pesquisa.bvsalud.org/portal/resource/pt/biblio-970871 
Galvão, C. M., Mendes, K. D. S. \& Silveira, R. C. C. P. (2010). Revisão integrativa: método de revisão para sintetizar as evidências disponíveis na literatura. In: Brevidelli, M. M., Sertório, S. C. M. Trabalho de conclusão de curso: guia prático para docentes e alunos da área da saúde. 105-26. https://pesquisa.bvsalud.org/portal/resource/pt/biblio-970871

Gavine, A., Macgillivray, S., Renfrew, M. J., Siebelt, L., Haggi, H. \& Mcfadden, A. (2017). Education and training of healthcare staff in the knowledge, attitudes and skills needed to work effectively with breastfeeding women: a systematic review. International Breastfeeding Journal, 12(6). https://www.scielo.br/scielo.php?pid=s0104-07072008000400018\&script=sci_abstract\&tlng=pt

Kologeski, T. K., Strapasson, M. R., Schneider, V., \& Renosto, J. M. (2017). Contato pele a pele do recém-nascido com sua mãe na perspectiva da equipe multiprofissional. Revista de Enfermagem UFPE online, v. 11(1):94-101. https://periodicos.ufpe.br/revistas/revistaenfermagem/article/view/11882

Leite, M. F. F. S., Barbosa, P. A., Olivindo, D. D. F. \& Ximenes, V. L. (2016). Promoção do aleitamento materno na primeira hora de vida do recém-nascido por profissionais da enfermagem / Promotion of breastfeeding in the newborn's first hour of life by nursing professionals. Arquivos de Ciências da Saúde UNIPAR , 20(2). https://pesquisa.bvsalud.org/portal/resource/pt/biblio-1300

Maroja, M. C. S., Silva, A. T. M. C. \& Carvalho, A. T. (2014). Iniciativa Hospital Amigo da Criança: uma análise a partir das concepcões de profissionais quanto às suas práticas. Revista Portuguesa de Saúde Pública, 32(1), 3-9. https://doi.org/10.1016/j.rpsp.2014.02.002

Matos, T. A., Souza, M. S., Santos, E. K. A., Velho, M. B., Seibert, E. R. C. \& Martins, N. M. (2010). Contato precoce pele a pele entre mãe e filho: significado para mães e contribuições para a enfermagem. Revista Brasileira de Enfermagem, 63(6): 998-1004. https://doi.org/10.1590/S003471672010000600020

Mendes, K. D. S., Silveira, R. C. C. P. \& Galvão, C. M. (2008). Revisão integrativa: método de pesquisa para a incorporação de evidências na saúde e na enfermagem. Texto Contexto Enfermagem, Florianópolis, 17(4), 758-64. https://doi.org/10.1590/S0104-07072008000400018.

Moher, D., Liberati, A., Tetzalaff, J. \& Altman, D. G. (2015). Preferres reporting items for systematic reviews and meta-analyses: the PRISMA statment. PLoS Medicine, 6(7). https://doi.org/10.1371/journal.pmed.1000097

Nuaimi, K. Al., Ali, R. \& Ali, F. H. (2019). The effect of a breastfeeding educational workshop on clinicans' knowledge, attitudes and practices. British Journal of Midwifery, 27(4). https://doi.org/10.12968/bjom.2019.27.4.242

Organização Mundial da Saúde - OMS. (2017). Aleitamento materno nos primeiros anos de vida salvaria mais de 820 mil crianças menores de cinco anos em todo o mundo. Brasília. https://www.paho.org/bra/index.php?option=com_content\&view=article\&id=5729:aleitamento-materno-nos-primeiros-anos-de-vidasalvaria-mais-de-820-mil-criancas-menores-de-cinco-anos-em-todo-o-mundo \&Itemid=820

Oxford. Centre for Evidence-based Medicine: levels of evidence (March 2009). http://www.cebm.net/oxford-centre-evidence-based-medicine-levels-evidencemarch-2009

Pereira, C. R. V. R., Fonseca, V. M., Oliveira, M. I. C., Souza, I. E. O. \& Mello, R. R. (2013). Avaliação de fatores que interferem na amamentação na primeira hora de vida. Revista Brasileira de Epidemiologia, 16, 525-34. https://doi.org/10.1590/S1415-790X2013000200026

Pernambuco. Humanização do parto. (2015). Nasce o respeito: informações práticas sobre seus direitos / Organização, Assessoria Ministerial de Comunicação. Comitê Estadual de Estudos de Mortalidade Materna de https://www.mppe.mp.br/mppe/attachments/article/4240/cartilha\%20humanizacao\%20do\%20parto\%20pdf.pdf

Pompeo, D. A., Rossi, L. A., \& Galvão, C. M. (2009). Revisão integrativa: etapa inicial do processo de validação de diagnóstico de enfermagem. Acta Paulista de Enfermagem, 22(4):434-8. https://doi.org/10.1590/S0103-21002009000400014.

Rodrigues, C. F., Poblete, M., Lipinski, J., \& Zamberlan, C. (2020). Práticas atuais de amamentação na primeira hora de vida em uma maternidade de risco habitual. Revista Eletrônica Acervo Saúde, 12(1), e1826. https://doi.org/10.25248/reas.e1826.2020

Sampaio, À. R. R., Bousquat, A., \& Barros, C. (2016). Contato pele a pele ao nascer: um desafio para a promoção do aleitamento materno em maternidade pública no Nordeste brasileiro com o título de Hospital Amigo da Criança. Revista Epidemiologia e Serviços de Saúde, 25(2): 281-90. https://doi.org/10.5123/s1679-49742016000200007.

Silva, L. A. T., Fonseca, V. M., Oliveira, M. I. C., Silva, K. S., Ramos, E. G., \& Game, S. G. N. (2020). Profissional que assistiu o parto e amamentação na primeira hora de vida. Revista Brasileira de Enfermagem, 73(2). https://doi.org/10.1590/0034-7167-2018-0448

Strapasson, M. R., Fischer, A. C. S., \& Bonilha, A. L. L. (2011). Amamentação na primeira hora de vida em um hospital privado de Porto Alegre/RS: relato de experiência. Revista de Enfermagem - UFSM, 1(3), 489-496. https://doi.org/10.5902/217976922824

Thuler, A. C. M. C., Wall, M. L., \& Souza, M. A. R. (2018). Caracterização das mulheres no ciclo gravídico-puerperal e o incentivo à amamentação precoce. Revista enfermagem UERJ, 26: e16936. https://doi.org/10.12957/reuerj.2018.16936

United Nations. Sustainable Development Goals. (2015) < https://sdgs.un.org/ >

Vieira, T. O., Vieira, G. O., Giugliani, E. R. J., Mendes, C. M. C., Martins, C. C., \& Silva, L. R. (2010). Determinants of breastfeeding initiation within the first hour of life in a Brazilian population: cross-sectional study. BioMed Central Public Health, 10, 760. https://bmcpublichealth.biomedcentral.com/articles/10.1186/1471-2458-10-760

World Health Organization and the United Nations Children's Fund - UNICEF. (2020). Protecting, promoting and supporting breastfeeding: the Baby-friendly Hospital Initiative for small, sick and preterm newborns. Geneva. 